\title{
The prevalence of sexually transmitted diseases among prostitutes in Malaysia
}

\author{
S Ramachandran, Y F Ngeow
}

\begin{abstract}
The prevalence of sexually transmitted diseases was determined among 370 prostitutes in Kuala Lumpur, Malaysia. Chlamydial cervicitis $(26.5 \%)$ was detected more frequently than gonorrhoea (14.25\%) and was associated more often with pelvic inflammatory disease. Concurrent infections and asymptomatic infections were common. Seropositivity to hepatitis $B$ and syphilis were $66.3 \%$ and $13.6 \%$ respectively. Women under 20 years of age had significantly higher rates of infection with Chlamydia trachomatis and hepatitis $B$ virus than older women.
\end{abstract}

\section{Introduction}

In Malaysia, prostitutes have been most often cited as primary contacts for men seeking treatment in sexually transmitted disease (STD) clinics. This study was undertaken to determine the prevalence of major STDs among a prostitute population in the capital city of Kuala Lumpur. As there are little data on the epidemiology of STDs in Malaysia and prostitutes may be an important source of infection, it was felt that a study of this nature would help to determine the pattern of STDs in urban Malaysia. The information obtained would be useful in the formulation of policies for the control of STDs in the country.

\section{Materials and methods \\ Study population}

The study population was recruited from patients seen in a private clinic in Kuala Lumpur, Malaysia and included full time prostitutes as well as others who admitted to prostitution, such as social escorts and women working in bars, cabarets, massage parlours and other after-hours establishments. The women attended the clinic for gynaecological checkups, general medical complaints as well as for

Department of Medical Microbiology, Faculty of Medicine, University of Malaya, 59100 Kuala Lumpur, Malaysia

S Ramachandran, Y F Ngeow symptoms related to genital tract disease. Some were referred by symptomatic pimps. The purpose of the study and the requirement for additional laboratory tests were explained to each patient. Owing to time constraints, the number of patients investigated was limited to not more than 10 a day. Hence, the first 10 suitable patients for the day were chosen to be study subjects.

\section{Clinical examination}

From each patient, a full history was taken to include age, ethnic group, marital status, work experience, choice of sexual partners, place of practice, date of last exposure, symptoms in the patient and her partner, whether any form of protection was used and a history of past or self medication.

All study subjects, regardless of presenting complaints, were examined in the clinic by wet mount microscopy for the presence of clue cells, yeast and trichomonas. Urethral and endocervical smears were Gram-stained for intracellular Gram-negative diplococci. Patients with genital ulcers were examined by dark ground microscopy for treponemes. Specimens were collected from the endocervix, urethra, rectum and pharynx for gonococcal culture, from the endocervix for chlamydial antigen detection and from the posterior vaginal fornix for the isolation of candida. An additional swab was collected for Herpes simplex virus (HSV) isolation from patients with oral or anogenital ulcers or cervical lesions such as vesicles, ulcers, erosions or areas of friability and redness. Blood was obtained for hepatitis B and syphilis serology. All specimens for bacterial and fungal culture were plated directly onto selective media in the clinic. Gonococcal cultures were transported to the laboratory in a candle jar while specimens for HSV isolation were sent in an ice flask.

\section{Laboratory examination}

Neisseria gonorrhoeae was isolated on Modified Thayer Martin agar incubated in $6 \% \mathrm{CO}_{2}$ at $35^{\circ} \mathrm{C}$ for up to 96 hours. Gonococci were confirmed by Gramstaining, oxidase-positive reaction and a positive, coagglutination test (Phadebact Monoclonal GC Test, Pharmacia Diagnostics, Uppsala, Sweden). Beta-lactamase production was examined by the use of Nitrocefin (Oxoid, Basingstoke, England). 
For the diagnosis of chlamydial cervicitis, an amplified enzymeimmunoassay (IDEIA Chlamydia Test, Boots-Celltech Diagnostic Ltd, UK) was used.

Specimens for fungal culture were plated on Sabouraud's dextrose agar. Yeast-like colonies were differentiated by germ tube formation, sugar assimilation and sugar utilisation tests.

HSV was isolated in Primary Monkey Kidney cells and identified by fluorescent antibody staining (Syva Co, Palo Alto, CA, USA).

The serological tests carried out were: (a) for syphilis, Venereal Disease Research Laboratory test (VDRL, Bacto, Detroit, USA) and Treponema pallidum haemagglutination test (TPHA, Fujirebio, Tokyo, Japan); (b) for hepatitis B surface antigen (HBs) and antibody, a radioimmunoassay (AUSRIA II-125 and AUSAB, Abbott Laboratories Diagnostic Division, North Chicago, USA).

\section{Results}

Over a five month period from March to July 1988, a total of 400 visits by 370 patients were investigated. None of the patients asked to participate in the study refused consent. They were not required to stay away from work for any length of time during the study. For patients with repeat visits who had been given appropriate treatment for their infection, each visit more than a week after the previous one was regarded a separate incidence of infection. The majority $\left(93.3^{\circ} \%\right)$ of patients were Chinese; the rest being Indians, Filipinos, Malays and Europeans. Foreigners made up $3.75 \%$ of the total. The women's ages ranged from 15 to 48 years with an average of 24.4 years and $20 \%$ under 20 years of age. The length of time they have been in their high risk occupations ranged from $<6$ months to $>6$ years, with an average of about 3.8 years. Three hundred and thirty-seven $(91 \%)$ were full time prostitutes. Most of them worked within the capital city of Kuala Lumpur but at least $8.5 \%$ also entertained clients in neighbouring countries in Southeast Asia as well as in Hong Kong, Taiwan and Japan. All except $5 \%$ of the women had regular sex partners. While only $10 \%$ requested their clients to wear condoms, $60 \%$ required their regular sex partners to do so. Oral sex was commonly practised $(70 \%$ ) but only $1 \%$ would submit to anal intercourse. It was suspected that many resorted to self-medication but only five gave a definite history of having taken antibiotics before seeking medical advice. None admitted to or had clinical evidence of intravenous drug abuse. However, the use of minor tranquillisers and hypnotics like diazepam and triazolam was common.

About half $(51.5 \%)$ of the women presented with symptoms of genital tract infection, the commonest being vaginal discharge and signs and symptoms suggestive of salpingitis such as low abdominal pain,
Table 1 Clinical presentations

\begin{tabular}{lc}
\hline Presentation & No $(\%)$ \\
\hline Asymptomatic & $194(48 \cdot 5)$ \\
Vaginal discharge & $60(15 \cdot 0)$ \\
Pelvic inflammatory disease & $58(14 \cdot 5)$ \\
Anogenital ulcer & $41(10 \cdot 25)$ \\
Anogenital wart & $34(8 \cdot 5)$ \\
Others & $13(3 \cdot 25)$ \\
Total & $400(100)$ \\
\hline
\end{tabular}

fever, dyspareunia, adnexal swelling and pain on pelvic examination (table 1). Rates of infection with various disease agents are given in table 2 . Overall, $70.1 \%$ had at least one test positive and $21.7 \%$ had concurrent infections. From cervical swabs, Chlamydia trachomatis was detected more frequently than gonococci. Among those with gonorrhoea, $49 \cdot 1 \%$ also had chlamydial cervicitis. Gonococci were most frequently isolated from the urethra $(12.1 \%)$ and cervix $(11.8 \%)$. There were $13(3.9 \%)$ cases of pharyngeal gonorrhoea of which five occurred in the absence of infection at other sites sampled. Overall, $37(34.3 \%)$ of gonococcal isolates were penicillinase-producers (PPNG). The pharyngeal isolates had the lowest percentage of PPNG $(23 \cdot 1 \%)$. In three instances, both PPNG and nonPPNG were isolated from different sites in the same patient. Of the women presenting with pelvic inflammatory disease, $27 \cdot 6 \%$ had chlamydia and $15.5 \%$ had gonococci in their cervical swabs.

HSV was recovered from $16 / 41(39 \%)$ anogenital ulcers and $7 / 17(41 \cdot 2 \%)$ non-ulcerative cervical lesions. All the isolates except one were HSV type 2.

Serological evidence of exposure to Treponema pallidum and hepatitis B virus (HBV) was marked. When disease prevalence was related to age, women

Table 2 Prevalence of STDs among prostitutes in Malaysia

\begin{tabular}{lcc}
\hline Test & No tested & No $(\%)$ positive \\
\hline Gonococcal culture & 400 & $57 \star(14 \cdot 25)$ \\
Chlamydial EIA & 400 & $106(26 \cdot 5)$ \\
Candidal culture & 397 & $139(35 \cdot 0)$ \\
HSV isolation & $58 \dagger$ & $23(39 \cdot 7)$ \\
Wet film microscopy & & \\
$\quad$ for T vaginalis & 400 & $38(9 \cdot 5)$ \\
D-G microscopy for & & $1(2 \cdot 4)$ \\
$\quad T$ pallidum & 41 & $17(7 \cdot 1)$ \\
VDRL & 238 & $32(13 \cdot 6)$ \\
TPHA & 236 & $6(6 \cdot 1)$ \\
HBs antigen & 98 & $65(66 \cdot 3)$ \\
Anti-HBs & 98 & $34(8 \cdot 5)$ \\
Genital warts $\ddagger$ & 400 &
\end{tabular}

*No of patients with positive gonococcal culture from any site. Multiple isolates from the same patient were counted as one.

tOnly patients with cervical lesions and anogenital ulcers were swabbed for HSV isolation.

†clinical diagnosis.

$\mathrm{D}-\mathrm{G}=$ dark-ground 
under 20 years were found to have significantly higher rates of infection with chlamydia and HBV $(\mathrm{p}<0.05)$.

\section{Discussion}

In Malaysia, it is illegal to organise prostitution or to live on the immoral earnings of another person but voluntary prostitution by adult non-Muslims is not a crime. Nevertheless, prostitution is regarded a social menace and prostitutes have always been blamed for transmitting STDs to their clients. While some high class call girls do voluntarily seek medical check-ups, the majority of prostitutes are either ignorant of the need for regular medical attention or are unwilling to pay for it.

In this study, we examined the prevalence of major STDs among prostitutes and compared them with available data for other groups of patients. Among 750 consecutive antenatal patients (37\% Chinese) at the University Hospital, Kuala Lumpur, only $0.54 \%$ were found to have cervical gonorrhoea. ${ }^{1}$ In a similar setting, $2 \%$ were positive for chlamydial antigen, $22 \%$ for yeasts and $2 \%$ for trichomonas (Y F Ngeow, unpublished data). Out of 4426 Chinese pregnant women, $0.93 \%$ were VDRL positive and $0.36 \%$ TPHA reactive. ${ }^{2}$ These rates were all much lower than those found for prostitutes. However, when the data for men and non-prostitute women attending STD clinics were examined, it was found that of 517 men with urethritis (all of whom admitted to extramarital intercourse, mostly with prostitutes), $37.9 \%$ had gonorrhoea and $33.1 \%$ were positive for chlamydial antigen by enzymeimmunoassay. Among 120 women, $67 \%$ of whom were contacts of symptomatic men, $13.3 \%$ had gonorrhoea and $35 \%$ had chlamydial cervicitis. Syphilis seropositivity for these men and women was $20.6 \%$ and $20.7 \%$ for VDRL and $29 \%$ and $28.7 \%$ for TPHA respectively. From these data, it would appear that promiscuous men and their regular sex partners form an even larger pool of infection than prostitutes. It should be kept in mind, however, that the men were all symptomatic and the women were contacts of symptomatic men whereas the prostitutes were chosen at random and almost $50 \%$ had no signs and symptoms of genital tract infection at the time of examination. On the other hand, $31.6 \%$ and $34.9 \%$ of the prostitutes with gonorrhoea and chlamydial cervicitis respectively were asymptomatic. Hence the prostitutes are a good example of a core-group of transmitters with high prevalence of asymptomatic infection who are responsible for the sustained transmission of STDs in a community.

Among Malaysian Chinese women with one or no sex partners, the HBV infection rate was found to be $40.7 \%$ (CY Gan, unpublished data). The higher infection rate among our prostitutes (66.3\%) supported heterosexual transmission of $\mathrm{HBV}$. The HBs carriage $(6.1 \%)$, however, was within the range $(4.7 \%-8.4 \%)$ reported for Chinese antenatal women. ${ }^{3}$ This was consistent with the observation by others that persistent HBs antigenaemia was more often related to exposure at an early age and could reflect not just an increased likelihood of infection but a diminished ability to clear the virus after an infection through a specific defect in the immune response to $\mathrm{HBV}$ infection. ${ }^{4}$

The data presented in this study show that chlamydial genital infections have become more common than gonorrhoea among our STD clinic patients and that chlamydia is probably more important than gonococci in the aetiology of pelvic inflammatory disease.

With international travel, it is not surprising that the pattern of STDs in Malaysia is similar to that in many Western and neighbouring countries. The prime concern among STD health workers at the moment is the heterosexual transmission of the human immunodeficiency virus (HIV). Sixty of the women in this study gave consent for anti-HIV serology. Fortunately, none were positive. In Malaysia, HIV infections have been associated with mainlining drug addicts, haemophiliacs and homosexual men with overseas sexual contact but not yet with prostitutes. The fear of acquired immunodeficiency syndrome has helped to focus attention on the control of STDs in general. In this respect, an important consideration would be the large reservoir of infection among promiscuous groups such as prostitutes and the men who patronize them.

The authors thank Associate Professor S F Yap (Department of Pathology, University of Malaya) for help with hepatitis serology and Professor S K Lam (Department of Medical Microbiology, University of Malaya) for his help with HSV isolation. We also thank Lederle Laboratories, Cyanamid (Far East) Ltd, and Pfizer (Malaysia) Sdn Bhd for funding this study.

Address for correspondence: Y F Ngeow, Department of Medical Microbiology, Faculty of Medicine, University of Malaya, 59100 Kuala Lumpur, Malaysia.

1 Goh TH, Ngeow YF, Teoh SK. Screening for gonorrhoea in a South-East Asian prenatal clinic. Sex Transm Dis 1981;8:67.

2 Goh TH, Ngeow YF. Serological screening for syphilis during pregnancy in a multiethnic Asian population. Asia-Oceania $J$ Obstet Gynaecol 1989;15:67-70.

3 Tan PE. Antenatal screening for Hepatitis B in pregnant women. Malaysian J Pathol 1988;10:79-83.

4 Editorial. Immunization against Hepatitis B. $\mathrm{Br}$ Med J 1980;281:1585-6.

Accepted for publication 26 July 1990 